\title{
Éditorial : Les nouveaux enjeux des migrations en Belgique
}

Editorial: New Migration Issues in Belgium

Editorial: Nuevos temas de migración en Bélgica

Marco Martiniello, Jacinthe Mazzocchetti et Andrea Rea

\section{(2) OpenEdition}

Édition électronique

URL : https://journals.openedition.org/remi/6369

DOI : $10.4000 /$ remi.6369

ISSN : $1777-5418$

Éditeur

Université de Poitiers

Édition imprimée

Date de publication : 1 juin 2013

Pagination : 7-14

ISBN : 979-10-90426-08-5

ISSN : 0765-0752

\section{Référence électronique}

Marco Martiniello, Jacinthe Mazzocchetti et Andrea Rea, «Éditorial : Les nouveaux enjeux des

migrations en Belgique », Revue européenne des migrations internationales [En ligne], vol. 29 - n² | 2013, mis en ligne le 01 juin 2016, consulté le 14 avril 2022. URL : http://journals.openedition.org/remi/6369 ; DOI : https://doi.org/10.4000/remi.6369 


\title{
Éditorial
}

\section{Les nouveaux enjeux des migrations en Belgique}

\author{
..o Marco Martiniello', Jacinthe Mazzocchetti ${ }^{2}$ \\ et Andrea Rea ${ }^{3}$
}

L'objectif de ce numéro n'est pas de retracer la longue histoire migratoire de la Belgique, petite démocratie ${ }^{4}$ coincée entre des grandes nations de la vieille Europe, mais de faire le point sur les nouvelles migrations et les nouveaux enjeux migratoires depuis les années 1990. Depuis lors, les données en termes de flux migratoires (rapport entre les entrées et les sorties) montrent une augmentation constante des étrangers, et ce en dépit de la crise économique qui sévit dans le pays. La présence des étrangers est ainsi plus élevée actuellement que durant la période reconnue comme celle de l'âge d'or de l'immigration qui va de 1946 à 1974. Cependant les types de migrations récentes se sont transformés et diversifiés.

On observe une augmentation considérable de migrants en provenance des pays de l'Europe de l'Est, et notamment des nouveaux États membres de l'Union européenne (CECLR, 2012). En effet, en 2009, I'immigration en provenance des pays européens (UE-27) représentait $63 \%$ des migrations totales. Au sein de ce groupe, le changement majeur concerne l'arrivée de migrants qui viennent des douze nouveaux États membres de I'UE, $19 \%$ contre $34 \%$ aux ressortissants de I'UE-15 et $10 \%$ aux ressortissants européens en dehors de I'UE (y compris la Turquie). De ce point de vue, le changement le plus important en matière d'immigration réside dans l'augmentation des migrants de I'UE. Le nombre des immigrés provenant des nouveaux États membres de I'UE a particulièrement crû entre 2004 et 2009, passant de 7 à $19 \%$ de la population étrangère. Les Polonais,

\footnotetext{
1 Directeur de recherche au FNRS, Professeur de sociologie à I'Université de Liège, Directeur du Centre d'Étude de I'Ethnicité et des Migrations (CEDEM), boulevard du Rectorat 7, B-4000 Liège, Belgique ; M.Martiniello@ulg.ac.be

2 Professeur d'anthropologie, Université catholique de Louvain, Membre du Laboratoire d'Anthropologie Prospective (LAAP), Place Montesquieu 1, B-1348 Louvain-la-Neuve, Belgique ; jacinthe.mazzocchetti@uclouvain.be

3 Professeur de sociologie, Université Libre de Bruxelles (ULB), Groupe de recherche sur les Relations Ethniques, les Migrations et I'Égalité (GERME), avenue Jeanne 44, CP124, B-1050 Bruxelles, Belgique; area@ulb.ac.be
}

4 La Belgique est à la fois un pays d'immigration, d'émigration et de transit depuis des siècles, bien avant sa constitution en tant qu'État indépendant en 1831. 
les Roumains et les Bulgares constituent la majorité de ces nouveaux arrivants, alors qu'ils étaient pratiquement absents des statistiques avant 2000. Parmi les ressortissants des pays non-européens figurent trois nationalités de l'immigration traditionnelle en Belgique, le Maroc, la République Démocratique du Congo, la Turquie, mais aussi de nouveaux pays d'origine: Cameroun, Guinée, Brésil et Chine.

Au 1er janvier 2011, la Belgique comptait 10951266 habitants, dont 1119256 étaient de nationalité étrangère, soit $10,2 \%$ de la population. II existe toutefois de grandes disparités entre les trois régions géographiques et politiques belges. Ainsi, dans la région bruxelloise, les étrangers représentent $31,4 \%$ de la population totale alors que cette proportion est de 6,7 \% en Flandre, où la part des étrangers a cru ces dix dernières années, et de 9,6\% en Wallonie, où la part des étrangers diminue régulièrement. Les ressortissants de I'UE représentent $66,85 \%$ de la population étrangère totale en 2011. Les Italiens, les Français et les Hollandais représentent $40 \%$ de cette population. Cependant, la plus forte progression concerne les Polonais, les Roumains et les Bulgares. Entre 2007 et 2011, ces groupes nationaux ont augmenté respectivement de $53,3 \%, 69,7 \%$ et $77,4 \%$.

En raison de leur statut de citoyens européens et du droit à la libre circulation, encore qu'elle soit partielle du fait de l'obligation de disposer d'un permis de séjour pour entrer sur le marché du travail, les nouveaux ressortissants européens ne sont pas considérés comme des immigrés. Cette catégorie semble être réservée dans les forums médiatiques et politiques, et parfois scientifiques, aux seuls étrangers provenant des pays non-européens. Cette manière de rendre compte de l'immigration conduit à une réduction analytique qui rend difficile la compréhension des processus migratoires contemporains, et tout particulièrement des dynamiques sur le marché du travail. En effet, souvent, l'analyse de l'immigration se centre sur le mode d'application de la législation relative à I'accès et au séjour sur le territoire réduisant de la sorte l'immigration aux types et aux nombres de permis de séjour délivrés.

L'approche en termes de motifs légaux d'attribution des titres de séjours ne donne qu'une vue partielle des nouvelles migrations, dans la mesure où elle ne concerne que les ressortissants des États tiers. Pour ceux-ci, la situation est proche des pays limitrophes de la Belgique. Ainsi, sur les 67653 premiers titres de séjour octroyés en 2010, le regroupement familial représente 42,4\%. La deuxième source d'attribution de séjour relève des raisons humanitaires ou médicales (17,7\%). Dans cette catégorie figurent notamment les régularisations au cas par cas de migrants. Comparativement aux pays comme la France, l'Allemagne et les Pays-Bas, l'immigration étudiante est relativement faible $(8,4 \%)$ et plus encore l'immigration pour raison de travail qui est de $6,1 \%$ alors que cette proportion est de 19,2 \% aux Pays-Bas, 14,1 \% en Allemagne et 9,3\% en France (CECLR, 2012 : 21). Toutefois, les motifs d'immigration varient fortement selon les nationalités. Si le regroupement familial est remarquable chez les Marocains et lesTurcs, les raisons humanitaires concernent majoritairement des personnes provenant de la RDC, de l'Arménie, de la Serbie, du Brésil et de la Russie.

L'évolution de la part des étrangers, en distinguant les ressortissants de I'UE et les ressortissants des pays tiers, nécessite de tenir compte de la plus grande disposition des seconds à avoir plus fréquemment recours aux procédures d'acquisition de la nationalité belge. En somme, les ressortissants européens 
qui gardent leur nationalité d'origine restent plus souvent catégorisés comme étrangers, alors que les étrangers ayant acquis la nationalité disparaissent des statistiques. Au 1er janvier 2011, parmi les personnes devenues belges, $68 \%$ sont d'origine non-UE (441 789) et $32 \%$ d'origine de I'UE (207 478). Plus précisément, pour les étrangers marocains, turcs et congolais, plus de $60 \%$ des migrants ont opté pour la nationalité belge (CECLR, 2012). Ces trois pays partagent une histoire particulière avec la Belgique : celle des migrations de main-d'œuvre (Maroc et Turquie) et celle liée au passé colonial (République Démocratique du Congo). La répartition globale de la population en 2011 était composée de $83 \%$ de Belges à la naissance, $10 \%$ d'étrangers et $8 \%$ d'étrangers devenus Belges.

Outre l'européanisation des migrations, la féminisation tendancielle des flux migratoires est aussi perceptible (Timmermans et al., 2012). L'augmentation du nombre des femmes n'est pas stable dans le temps et surtout ne concerne pas de façon équivalente toutes les nationalités d'origine. Jusqu'en 1993, en Belgique, il y avait entre 112 et 120 immigrations d'hommes pour 100 de femmes. La féminisation s'observe dans un premier temps entre 1994 et 1999 avant de redescendre et de repartir à la hausse entre 2003 et 2004 et puis après 2008 . Parmi les pays tiers, la proportion de femmes est de 48,96 \% alors qu'elle est de 49,48\% pour les pays de I'UE. Toutefois, des disparités importantes existent. Ainsi, chez les Philippins, les femmes sont largement majoritaires $(74,6 \%)$, ce qui est une caractéristique de cette nationalité. II en va de même, mais dans une moindre mesure, pour les migrants d'Amérique latine. La proportion de femmes est toujours supérieure à celle des hommes : $61 \%$ pour les Brésiliens, $59 \%$ pour les Équatoriens, $65 \%$ pour les Péruviens et $59 \%$ pour les Colombiens. Cette féminisation est aussi observée parmi les nouveaux migrants provenant de Pologne, de Roumanie et de Bulgarie, du moins un peu avant l'adhésion de ces pays à I'UE. Après quoi la tendance s'est inversée. Par contre, la part des femmes reste très forte pour d'autres migrations en provenance d'Europe de l'Est, comme I’Ukraine (64\%), la Biélorussie (65\%) et la Russie (56 \%).

Cette nouvelle donne migratoire a suscité de nouveaux débats et donné lieu à des politiques publiques qui font l'objet des contributions rassemblées dans ce numéro. Les différents articles revisitent des questions traditionnelles telles que l'activité économique des nouveaux arrivants, les formes de participation citoyenne des migrants d'origine non-européenne et les politiques d'intégration. Ils abordent aussi un thème moins fréquemment traité, celui de la participation politique transnationale des émigrants belges. Ces contributions partagent une même approche : celle de l'agency des migrants sans sous-estimer cependant les effets des cadres institutionnels et structurels. Mais c'est d'abord la dimension " entrepreneur de soi " qui sert de fil conducteur à la plupart des articles.

Les femmes migrantes en provenance d'Équateur et du Brésil étudiées par Marie Godin et Any Freitas se présentent comme les "entrepreneurs " de leurs trajets migratoires. L'immigration est considérée comme une "opportunité " ou un " pari d'avenir " et leur départ est en partie un choix, qui se fait parfois contre l'avis de leurs familles et de leur entourage. Les commerçants béninois étudiés par Martin Rosenfeld sont des "entrepreneurs " à la fois sur le plan commercial et migratoire, inaugurant de nouvelles formes de circulation entre I'Afrique et I'Europe. De même les acteurs associatifs, les jeunes, migrants et fils/filles de migrants (Afrique subsaharienne) étudiés par Nicole Grégoire et 
Jacinthe Mazzocchetti se posent également en tant qu' " entrepreneurs de soi " et " entrepreneurs d'identité ".

\section{Figures du travailleur migrant et carrières migratoires}

L'article d'Andrea Rea décrit la fragmentation des statuts d'emploi et la sédimentation des nationalités. Cette fragmentation participe d'un contexte plus global de mise en concurrence des " précaires " dans un contexte économique difficile, auquel s'ajoutent des dimensions ethniques et raciales. L'auteur propose également une analyse socio-historique de la transformation de la figure du travailleur migrant et de ses ambiguïtés : persistance dans la manière dont elle est pensée (imaginaire de la non-durabilité, consigné à certaines tâches, exécutant le "sale boulot ", etc.) et changement de contexte économique, social et politique. II montre comment l'élargissement de I'UE a conduit à une internalisation des bassins de recrutement des nouveaux travailleurs migrants. Penser le travail du migrant suppose aussi de prendre en compte la perte partielle du contrôle étatique des régulations du marché du travail que les discours et les pratiques des migrations temporaires du travail et des circulations des services viennent renforcer. Comme le soutiennent Ilke Adam et Marco Martiniello dans leur article qui porte sur les politiques d'intégration, toute analyse suppose a minima une intégration de l'échelle européenne et ne peut se conduire uniquement dans la cadre des États-nations. C'est particulièrement ce que met en exergue Andrea Rea lorsqu'il qualifie de délocalisations intérieures les pratiques de détachement de personnel liées à la libre circulation des services auxquelles ont de plus en plus souvent recours les entreprises lorsqu'il s'agit de recruter des travailleurs provenant des nouveaux États membres de l'Union européenne. Ces délocalisations intérieures s'ajoutent aux délocalisations sur place (Terray, 1999) caractérisant le travail informel des sans-papiers. Ceci permet de recourir à une "immigration sans travailleurs immigrés" (Rea, 2006). Ces travailleurs ne sont plus liés à l'État dans lequel ils travaillent temporairement, mais à l'entreprise qui les embauche. Par ailleurs, l'étude de l'évolution du contexte économique, de la dérégulation du marché du travail et de l'affectation des travailleurs démontrent combien certains segments du marché du travail où se trouvent concentrés les nouveaux migrants (horticulture, construction, domesticité) fonctionnent selon le principe des chaises musicales : un nouvel arrivant prend la place de celui qui s'est déplacé vers des postes de travail mieux payés et mieux reconnus.

Deux études de cas auprès de migrants hors-Europe viennent compléter I'analyse du paysage belge des nouvelles migrations de travail. Marie Godin et Any Freitas s'intéressent aux parcours de femmes migrantes venues d'Amérique latine (en particulier, du Brésil et de l'Équateur), insérées dans le secteur de la domesticité informelle et/ou en "zones grises " à Bruxelles. L'approche en termes de " carrière migratoire " (Martiniello et Rea, 2011) leur permet de combiner trois niveaux d'analyse : les caractéristiques individuelles des acteurs (niveau micro), la mobilisation des ressources et du réseau social (niveau méso) et la structure des opportunités et des contraintes des migrations internationales (niveau macro). Martin Rosenfeld s'intéresse aux entrepreneurs transnationaux béninois dans le secteur du commerce de voiture à partir d'un terrain multi-situé 
qui consiste à analyser les différents lieux et temps de ces échanges commerciaux transnationaux (Bruxelles-Cotonou). Bien qu'adoptant des méthodologies sensiblement différentes, ces deux contributions combinent analyses contextuelles et points de vue des acteurs et, dès lors, mettent en évidence les singularités des trajectoires et carrières migratoires en interrelation avec des histoires migratoires, des politiques et des réseaux sociaux différenciés.

Un des apports importants du texte de Marie Godin et Any Freitas est d'échapper à une vision victimisante d'une oppressante " chaîne du care global " en accordant une place majeure au changement et aux bifurcations possibles, à I'hétérogénéité des trajectoires. L'analyse des carrières migratoires, offre la possibilité de conjuguer les dimensions objectives (parcours juridico-institutionnel et socio-économique) et subjectives (évolution des identités, projets et statuts) et permet de mieux saisir la façon dont les conceptions " de soi " et " des possibles " des femmes migrantes évoluent en fonction des transformations du contexte économique, juridique, politique et social. Martin Rosenfeld met en évidence l'insuffisance du modèle d'immigration-intégration pour décrire de nouvelles pratiques migratoires et notamment ce qui est du ressort de l'ethnic entrepreneurship. Ainsi le commerce euro-africain d'exportation de véhicules d'occasion présente la particularité d'être une activité commerciale mise en œuvre par des migrants et exclusivement destinée à d'autres migrants, ce qui permet à l'auteur de critiquer le stéréotype associé aux migrants sub-sahariens " censés venir prendre d'assaut le système social de l'Europe forteresse ". D'une part, ces migrants investissent des sommes conséquentes en Belgique et d'autre part, n'ont aucunement l'intention de s'installer sur le territoire belge.

Enfin les articles d'Andrea Rea, Marie Godin et Any Freitas et Martin Rosenfeld nous livrent une analyse des circulations de personnes en interdépendance avec les politiques migratoires et législatives belges et européennes. Ils décrivent les " niches ethniques ", les jeux de chaises musicales et les possibilités d'insertion socio-économique en relation avec les politiques européennes à l'égard des ressortissants de I'UE et des migrants hors espace européen en matière notamment d'obtention de visas. Marie Godin et Any Freitas mettent en évidence les liens entre la migration latino-américaine, en particulier féminine et d'une part, l'augmentation de la demande dans le secteur de la domesticité et d'autre part, la possibilité d'entrer légalement en Europe, en détenant un visa touristique. Martin Rosenfeld décrit une situation bien différente, celle des ressortissants d'Afrique contraints d'organiser leur filière commerciale en fonction des restrictions d'octroi de visa Schengen. Sources de frustrations et de rancœurs, ces contraintes administratives poussent une série d'entrepreneurs à rediriger leur commerce en direction des États-Unis où les passeports pour raison commerciale s'obtiennent plus facilement.

\section{Intégration, citoyenneté et participation/expression (politique, rue)}

Une deuxième série d'articles traitent des politiques d'intégration des nouveaux migrants, mais prêtent aussi attention à ceux qui sont installés de longue date, ainsi qu'aux dynamiques de participation. 
Ilke Adam et Marco Martiniello, après avoir rappelé les particularités de I'État multinational (ou national contesté) belge, analysent les divergences et convergences des modèles et des politiques d'intégration en Belgique à partir d'une étude de cas concernant les parcours d'intégration pour immigrés, obligatoires en Flandre et, non centralisés et non obligatoires, du côté francophone. Les auteurs abordent la question du contexte historique et politique qui préside à l'élaboration des politiques d'intégration dans une Belgique qualifiée " de fédéralisme de désunion " (Swenden et Jans, 2006 : 877-894). Ils exposent les éléments contextuels saillants relatifs aux possibilités d'organisation et de participation des communautés migrantes en Belgique, mais aussi des émigrés belges dans le monde, question que discute Jean-Michel Lafleur dans son article. Premièrement, les débats entre communautés francophone et flamande, laissent peu de place à d'autres types de revendications communautaires, deuxièmement, les politiques et les législations en direction des migrants installés en Belgique, mais aussi destinées aux ressortissants belges vivant à l'étranger, sont bien plus discutées dans un face-à-face Flamands-Wallons, en lien avec des enjeux électoraux communautaires, qu'en réponse à des demandes et des revendications faites par ces groupes migrants de l'intérieur ou de l'extérieur.

Afin de comprendre la situation belge, Ilke Adam et Marco Martiniello soulignent " l'importance de la prise en considération du nationalisme minoritaire (Adam, 2011) ou du "multinational politics" (Jacobs, 2000 ; Martiniello, 2012), catégorie plus large incluant les réactions des francophones à ce nationalisme flamand, dans l'explication de plusieurs particularités des politiques d'intégration ". Ils en dégagent deux facteurs explicatifs fondamentaux des politiques d'intégration différenciées entre la Flandre et la Wallonie : la politisation divergente de l'immigration et de l'intégration et la force du nationalisme minoritaire flamand. Ainsi, écrivent-ils : " la position de l'électeur à l'égard des immigrés et de la question de l'intégration est, en Flandre plus qu'en Belgique francophone, devenue un élément déterminant dans le choix des électeurs ". Cette plus forte " politisation de l'immigration en Flandre par rapport à la Belgique francophone, due à la présence d'un parti d'extrême droite anti-immigré, ne peut être isolée du processus de construction de la nation sous-étatique flamande ". Processus inexistant côté francophone. Et si dans les derniers mois de 2012, un changement d'attitudes à l'égard des politiques d'intégration, notamment le fait de rendre obligatoire et effectif les parcours d'intégration, se laisse voir en Wallonie, les auteurs l'attribuent plutôt à la normalisation de ces parcours à travers les politiques européennes de l'intégration et la mise en œuvre de tels programmes dans les pays voisins.

II ressort de leurs analyses une certaine prise en étau des migrants. Les logiques qui président à la mise en place de politiques les concernant sont en définitive bien plus influencées par la résolution de problèmes politiques internes, plutôt que par des débats avec les intéressés sur les questions d'intégration. Dans ce contexte, I'article de Nicole Grégoire et de Jacinthe Mazzocchetti, relatif aux ressortissants d'Afrique subsaharienne, pose la question des possibilités d'existence et de reconnaissance des minorités en Belgique, des espaces et des moyens dont ils disposent pour faire entendre leur voix. De même que les " entrepreneurs en migration " rencontrés par Godin et Freitas et les " entrepreneurs transnationaux " rencontrés par Rosenfeld, les associations, les groupes de jeunes et les artistes des minorités ethnoculturelles, analysées par nos 
auteures, se présentent comme des "entrepreneurs de soi ". Face aux failles ou aux faillites des politiques d'intégration, il s'agit de lutter contre les assignations identitaires et de proposer dans l'agir d'autres manières de concevoir le " vivre ensemble " dans un monde cosmopolite.

Nicole Grégoire et Jacinthe Mazzocchetti ont mené un terrain auprès d'élites associatives "panafricaines " et auprès de groupes de jeunes s'auto-nommant " blacks ". Un des apports de ce regard croisé sur deux générations, migrants et fils/filles de migrants, concerne la diversité des réponses collectives apportées par la " diaspora africaine " de Belgique à la situation d'impuissance relative à laquelle elle doit globalement faire face. Elles analysent l'articulation complexe entre la double condition de " citoyen " et " d'autre ", souvent vécue comme antinomique dans les interactions quotidiennes. Situation commune aux deux générations, qui cependant y répondent de manière différente. Du côté de la génération des "parents ", qui ont généralement eux-mêmes migré, le combat fût d'abord d'asseoir la condition d' "immigré " afin de bénéficier d'une attention et d'une reconnaissance vis-à-vis des problèmes rencontrés dans la société d'accueil. Dans le monde associatif, notamment panafricains, les acteurs ont engagé une lutte pour la reconnaissance suivant une grammaire conjuguant altérité vécue, altérité affirmée et égalité statutaire. De leur côté, les jeunes, membres des dites "bandes urbaines ", mettent en avant une citoyenneté " indistinctive " et " non spécifique ". Ces jeunes ne se disent pas culturellement différents, mais assignés à la différence. Contrairement à leurs parents, c'est uniquement leur vécu de " racialisation " qui est constitutif de leur identité collective affirmée. S'ils se regroupent par " affinité ethno-raciale " et puisent alors dans un imaginaire transnational, leurs aspirations ne s'orientent pas vers un autre horizon que celui de la société qui les a vus naître et/ou dans laquelle ils ont grandi.

En clôture de ce numéro, I'article de Jean-Michel Lafleur revient sur l'émigration des Belges à l'étranger et sur leur participation politique transnationale en tant que Belges expatriés. L'auteur montre bien, et en cela il rejoint les propos de certains des articles publiés dans ce numéro, l'interdépendance entre ce qui est mis en place au niveau juridique et politique, afin de permettre la participation politique des Belges expatriés, et les enjeux communautaires internes à la Belgique qui semblent primer sur les droits/revendications/demandes des émigrants eux-mêmes, d'ailleurs peu associés aux politiques les concernant ; " [...] il est incontestable que c'est bien la compétition électorale entre les différents partis politiques belges qui a déterminé I'issue de ces deux débats artificiellement réunis à la fin des années 1990 sur le droit de vote des citoyens européens et sur le droit de vote de la diaspora ". Le manque d'intérêt des partis politiques pour la question des droits politiques de la diaspora est notamment confirmé par le faible nombre de propositions législatives à ce sujet dans les années 1990, nous dit l'auteur. "Plus précisément, le cas belge semble démontrer que l'absence de mobilisation politique des émigrés permet à l'élite politique du pays d'origine d'instrumentaliser cette population pour servir ses objectifs sur la scène politique intérieure ". En outre, dans le cas belge, les intérêts économiques ne sont pas les seuls à encourager les États à adopter une approche instrumentale des relations avec la diaspora, relève l'auteur, insistant sur la nécessaire prise en compte des divisions ethnolinguistiques. 


\section{Références bibliographiques}

Adam Ilke (2011) Des approches différenciées de la diversité. Les politiques d'intégration des personnes issues de l'immigration en Flandre, en Wallonie et à Bruxelles, in Julie Ringelheim Éd., Les droits et la diversité culturelle, Bruxelles, Éditions Bruylant, pp. 253-300.

CECLR (2012) Migration, Bruxelles, Rapport annuel du Centre pour l'égalité des chances et la lutte contre le racisme.

Jacobs Dirk (2000) Multinational and Polyethnic Politics entwined: Minority Representation in the Region of Brussels-Capital, Journal of Ethnic and Migration Studies, 26 (2), pp. 289-304.

Martiniello Marco (2012) Belgium, in Christian Joppke and Leslie Seidle Eds., Immigrant Integration in Federal Countries, Montreal and Kingston, McGillQueen's University press, pp. 57-77.

Martiniello Marco et Rea Andrea (2011) Des flux migratoires aux carrières migratoires, SociologieS, Dossiers, Migrations, pluralisation, ethnicisation des sociétés contemporaines, [en ligne], mis en ligne le 18/10/2011. URL : http://sociologies.revues.org/3694

Rea Andrea (2006) L'immigration sans immigrés, in Stéphane Beaud, Joseph Confavreux et Jade Lindgaard Éds., La France invisible, Paris, La Découverte, pp. 439-442.

Swenden Wilfried and Jans Maarten Theo (2006) "Will it stay or will it go?" Federalism and the sustainability of Belgium, West European Politics, 29 (5), pp. 877-894.

Terray Emmanuel (1999) Le travail des étrangers en situation irrégulière ou la délocalisation sur place, in Étienne Balibar, Monique Chemillier-Gendreau, Jacqueline Costa-Lascoux et Emmanuel Terray Éds., Sans-papiers : I'archaïsme fatal, Paris, La Découverte, pp. 9-34.

Timmerman Christiane, Martiniello Marco, Rea Andrea et Wets Johan (Éds.) (2012) Femmes dans les processus migratoires contemporains, Gent, Academia Press, $314 \mathrm{p}$. 